\title{
Digitally Continuous Multivalued Functions, Morphological Operations and Thinning Algorithms
}

\author{
Carmen Escribano - Antonio Giraldo . \\ María Asunción Sastre
}

\begin{abstract}
In a recent paper (Escribano et al. in Discrete Geometry for Computer Imagery 2008. Leeture Notes in Computer Science, vol, 4992, pp. 81-92, 2008) we have introduced a notion of continuity in digital spaces which extends the usual notion of digital continuity. Our approach, which uses multivalued functions, provides a better framework to define topological notions, like retractions, in a far more realistic way than by using just single-valued digitally continuous functions.

In this work we develop properties of this family of continuous functions, now concentrating on morphological opcrations and thinning algorithms. We show that our notion of continuity provides a suitable framework for the basic operations in mathematical morphology: crosion, dilation, closing, and opening. On the other hand, concerning thinning algorithms, we give conditions under which the existence of a retraction $F: X \longrightarrow X \backslash D$ guarantees that $D$ is deletable. The converse is not true, in general, although it is in certain particular important cases which are at the basis of many thinning algorithms.
\end{abstract}

Keywords Digital space - Continuous function . Mathematical morphology $\cdot$ Simple point $\cdot$ Retraction . Thinning

C. Escribano - A. Giraldo (M) - M.A. Sastre

Departamento de Malemática Aplicada, Facultad de Informática, Universidad Politécnica, Campus de Montegancedo, Boadilla del Monte, 28660 Madrid, Spain

e-mail: agiraldo@fi.upm.es

C. Escribano

c-mail: cescribano@fi.upm,cs

M.A. Sastre

e-mail: masastre@ fi.upm.es

\section{Introduction}

The notion of continuous function is a fundamental concept in the study of topological spaces. For dealing with digital spaces, several approaches to define a reasonable notion of continuous function have heen proposed. The first one goes back to A. Rosenfeld [19] in 1986. He defined continuous functions in a way similar to that used for continuous maps in $\mathbb{R}^{n}$. It turned out that continuous functions agreed with functions taking 4-adjacent points into 4-adjacent points. $\mathrm{He}$ proved, amongst other results, that a function between digital spaces is continuous if and only if it takes connected sets into connected sets. Independently of Rosenfeld, L. Chen $[5,6]$ seems to have developed the same notion of continuity, using the tems immersion, gradually varied operator, and gradually varied mapping. Chen's work appeared originally in Chinese.

More results related to this type of continuity were proved by L. Boxer in [1] and, more recently, in [2-4]. In these papers, he introduced such notions as homeomorphism, retracts, and homotopies for digitally continuous functions, applying these notions to define a digital fundamental group, digital homotopies, and to compute the fundamental group of sphere-like digital images. However, as he recognizes in [3], there are some limitations with the homotopy equivalences he gets. For example, while all simple closed curves are homeomorphic and hence homotopically equivalent with respect to the Euclidean topology, in the digital case two simple closed curves can be homotopically equivalent only if they have the same cardinality.

A different approach was suggested by V. Kovalevsky in [17], using multivalued functions. This seems reasonable, sinec an expansion such as $f(x)=2 x$ must take 1 pixcl to 2 pixcls if the image of an interval has still to be connected. He calls a multivalued function continuous if the pre-image of 
an open set is open. He considers, however, that another important class of multivalued functions is what he calls "connectivity preserving mappings." By its proper definition, the image of a point by a connectivity preserving mapping is a connected set. This is not required for merely continuous functions. He finally asserts that the substitutes for continuous functions in finite spaces are the simple connectivity preserving maps, where a connectivity preserving map $f$ is simple if for any $x$ such that $f(x)$ has more than I element then $f^{-1} f(x)=\{x\}$. However, in this case it would be possible to map the center of a $3 \times 3$ square to the $k$-boundary of it leaving the points of the $k$-boundary fixed, obtaining in this way a "continuous" retraction from the square to its $k$-boundary, something impossible in the continuous realm.

The multivalued approach to continuity in digital spaces has also been used by R. Tsaur and M. Smyth in [23], where a notion of continuous multifunction for discrete spaces is introduced: A multifunction is continuous if and only if it is "strong" in the sense of taking neighbors into neighbors with respect to the Hausdorff metric. They use this approach to prove some results concerning the existence of fixed points for multifunctions. However, although this approach allows more flexibility in the digitization of continuous functions defined in continuous spaces, it is still a bit restrictive, as shown by the fact that the multivalued function used by them to illustrate the convenience of using multivalued functions is not a strong continuous multifunction.

In a recent paper [7] the authors presented a theory of continuity in digital spaces, using multivalued functions, which extends the one introduced by Rosenfeld and provides a framework to define topological notions, like relractions, in a far more realistic way than by using just single-valued digitally continuous functions. In particular, the deletion of simple points, one of the most important processing operations in digital topology, is characterized as a particular kind of retraction.

In this work we look more deeply into the properties of this family of continuous functions, now concentrating on morphological operations and thinning algorithms.

In Sect. I we revise the basic notions of digital topology required throughout the paper. In particular we recall different adjacency relations used to model digital spaces. In Sect. 2 we revise Rosenfeld's notion of digitally continuous function. In Sect. 3 we introduce the notion of subdivision of a topological space used to define continuity for multivalued functions and show some basic properties concerning the behavior of digitally continuous multivalued functions under restriction and composition. In Sect. 4 we show that the basic morphological operations of dilation and closing are continuous functions. We also show that, although the dual operations of erosion and opening cannot be modeled as continuous functions, they are so if we consider them defined on the set of white pixels. In Sect. 5 we show that the deletion of simple points can be completely characterized in terms of digitally continuous multivalued functions, and in Sect. 6 we extend this result to simple pairs. In the last sections we characterize thinning algorithms in terms of digitally continuous multivalued functions. Specifically, we show that the existence of an $(\mathcal{N}, k)$-retraction $F: X \longrightarrow X \backslash D$ guarantees that $D$ is 4-deletable (respectively, 8 -deletable) whenever $D$ is made of 4-boundary (respectively, 8 -simple) points. The converse is not true in general although it holds in certain particular important cases which are at the basis of many thinning algorithms.

For information on Digital Topology we recommend the survey [15] and the books by Kong and Rosenfeld [16], and by Klette and Rosenfeld [13]. Other results on the discretization of topological notions can be found in $[9,10]$. As an illustration of the usefulness of topological results, as those presented in this paper, in applications, we refer the reader to [11,21], where the combination of continuous and digital topological notions has allowed the development of algorithms to solve important problems, such as topological constrained segmentation.

\section{Digital Spaces}

In this section we recall the basic notions of digital topology. We consider $\mathbb{Z}^{n}$ as model for digital spaces.

Definition 1 Two points in the digital line $\mathbb{Z}$ are adjacent if their coordinates differ by a unit. Two points in the digital plane $\mathbb{Z}^{2}$ are 8 -adjacent if they are different and their coordinates differ in at most a unit. They are called 4-adjacent if they are 8-adjacent and differ in exactly one coordinate. Note that a point is 8-adjacent to 8 different points and is 4-adjacent to 4 different points. Two points of the digital 3space $\mathbb{Z}^{3}$ are 26 -adjacent if they are different and their coordinates differ in at most a unit. They are called 18-adjacent if they are 26 -adjacent and differ in at most two coordinates, and they are called 6-adjacent if they are 26-adjacent and differ in exactly one coordinate. In an analogous way, adjacency relations are defined in $\mathbb{Z}^{n}$ for $n \geq 4$, for example, in $\mathbb{Z}^{4}$ there exist 4 analogous adjacency relations: 80 adjacency, 64-adjacency, 32-adjacency and 8-adjacency.

Given $p \in \mathbb{Z}^{n}$ and $X \subset \mathbb{Z}^{n}$ we say that $p$ and $X$ are $k$ adjacent if $p \notin X$ and there exists $x \in X$ such that $p$ and $x$ are $k$-adjacent.

Given $X_{1}, X_{2} \subset \mathbb{Z}^{n}$ we say that $X_{1}$ and $X_{2}$ are $k$-adjacent if $X_{1} \cap X_{2}=\emptyset$ and there exist $x_{1} \in X_{1}$ and $x_{2} \in X_{2}$ such that $x_{1}$ and $x_{2}$ are $k$-adjacent.

Alternatively, the adjacency of a point and a set, or the adjacency between two sets, can be defined replacing the condition $X_{1} \cap X_{2}=\emptyset$ by $X_{1} \neq X_{2}$, or, like, for example, in [16], without imposing any of those conditions. The results in the paper and the proofs, with slight modifications, 

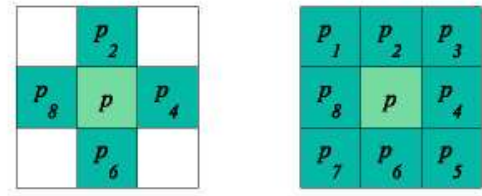

Fig. $1 \mathcal{N}_{4}(p)(l e f t)$ and $\mathcal{N}_{8}(p)($ right $)$ with labels

Fig. 2 A set with three 4-connected components and two 8-connected components

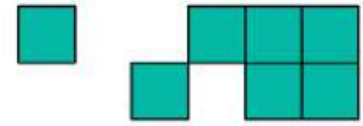

are still valid in both cases. However, in the context of this paper, it is more natural to require adjacent sets to be disjoint. The reason is that we will replace points by subdivisions of them (see Fig. 9). Since one point is not adjacent to itself, the set obtained after subdividing it, should not be adjacent to itself. Therefore, adjacent sets should be at least different. Moreover, if we consider a set $\{x, y\}$ formed by two non-adjacent points, then $\{x\}$ and $\{x, y\}$ are not adjacent sets in any definition and, hence, the sets obtained after subdividing them, although different and satisfying the definition in [16], should not be adjacent to each other.

Definition 2 If $p \in \mathbb{Z}^{2}$ we define $\mathcal{N}_{4}(p)$ and $\mathcal{N}_{8}(p)$ as in Fig. 1, i.e., $\mathcal{N}_{4}(p)$ is the set of points 4 -adjacent to $p$, while $\mathcal{N}_{8}(p)$ is the set of points 8 -adjacent to $p$, also denoted simply as $\mathcal{N}(p)$.

In $\mathbb{Z}^{3}$ there are three kinds of neighborhood: $\mathcal{N}_{6}(p)$, $\mathcal{N}_{18}(p)$, and $\mathcal{N}_{26}(p)$.

Definition 3 A $k$-path $P$ in $\mathbb{Z}^{2}(k \in\{4,8\})$ from the point $q_{0}$ to the point $q_{r}$ is a sequence of points $P=$ $\left\langle q_{0}, q_{1}, q_{2}, \ldots, q_{r}\right\rangle$ such that $q_{i}$ is $k$-adjacent to $q_{i+1}$, for every $i \in\{0,1,2, \ldots, r-1\}$. If $q_{0}=q_{r}$ then it is called a closed path.

A set $X \subset \mathbb{Z}^{2}$ is $k$-connected if for every pair of points of $X$ there exists a $k$-path contained in $X$ joining them. A $k$-connected component of $X$ is a maximal $k$-connected set (see Fig. 2).

Analogous definitions can be given for $\mathbb{Z}^{n}, n \geq 3$.

Definition 4 Given $X \subset \mathbb{Z}^{2}, k \in\{4,8\}$ and $p \in X$, we say, according to [16], that:

(i) $p$ is a $k$-isolated point of $X$ if $\mathcal{N}_{k}(p) \cap X=\emptyset$,

(ii) $p$ is a $k$-interior point of $X$ if $\mathcal{N}_{\bar{k}}(p) \subset X$,

(iii) $p$ is a $k$-boundary point of $X$ if $\mathcal{N}_{\bar{k}}(p) \cap\left(\mathbb{Z}^{2} \backslash X\right) \neq \emptyset$,

where $\bar{k}=4$ if $k=8, \bar{k}=8$ if $k=4$ (this notation will be used throughout the paper). We denote by $\partial_{k} X$ the $k$ boundary of $X$ (see Figs. 3 and 4).

Note that a point is $k$-interior if and only if it is not a $k$-boundary point.

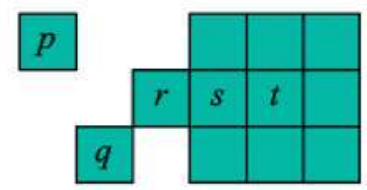

Fig. $3 p$ is 4-isolated and 8-isolated, $q$ is 4-isolated but not 8-isolated, $r$ is 4-boundary and 8-boundary, $s$ is 4-boundary but 8-interior, $t$ is both 4 -interior and 8 -interior
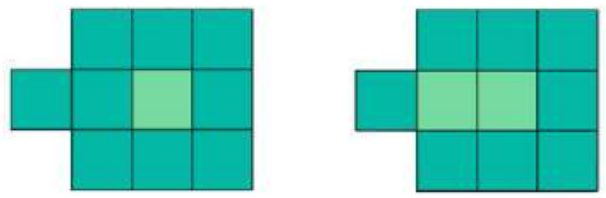

Fig. 4 Left: 4-boundary (dark) and 4-interior (light) of a set. Right: 8 -boundary (dark) and 8-interior (light) of the same set

Using these notions we can define the $k$-interior (respectively, $k$-boundary of $X$ ) as the set of points of $X$ which are $k$-interior (respectively, $k$-boundary) points of $X$.

\section{Digitally Continuous Single-Valued Functions}

In this section we revise the notion of digitally continuous function and some of its properties.

Definition 5 Let $f: X \subset \mathbb{Z}^{m} \longrightarrow \mathbb{Z}^{n}$ be a function between digital spaces with adjacency relations $k$ and $k^{\prime}$, respectively. According to [2, 19], $f$ is $\left(k, k^{\prime}\right)$-continuous if and only if $f$ sends $k$-adjacent points to the same point or to $k^{\prime}$-adjacent points. When $m=n$ and $k=k^{\prime}, f$ is said to be just $k$ continuous.

We will say that $f: X \subset \mathbb{Z}^{m} \longrightarrow \mathbb{Z}^{n}$ is continuous if it is $\left(k, k^{\prime}\right)$-continuous and the adjacencies $k$ and $k^{\prime}$ are understood.

Examples of digitally $k$-continuous functions are the identity, any constant function, translations $f(z)=z+r$, inversions $f\left(z_{1}, z_{2}\right)=\left(z_{2}, z_{1}\right), \ldots$. Another example is given by the following function taking a hollow square $S_{5}$ to a smaller one $S_{3}$.

The function which takes the corners of $S_{5}$ to the corresponding corners of $S_{3}$, and the points between two corners of $S_{5}$ to the point between the corresponding corners of $S_{3}$, is digitally $k$-continuous for $k \in\{4,8\}$ (see Fig. 5). However, for $k \in\{4,8\}$, there is not a digitally $k$-continuous function from $S_{3}$ to $S_{5}$ taking $a$ to $A, b$ to $B, c$ to $C$ and $d$ to $D$, since then it would not be possible to define the image of the rest of the points in such a way that $k$-adjacent points of $S_{3}$ are taken to the same point or to $k$-adjacent points of $S_{5}$ (see Fig. 6).

In [19] Rosenfeld stated and proved several results about digitally continuous functions related to operations with 

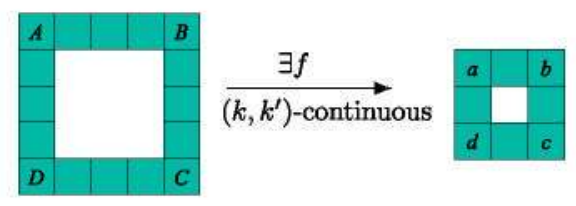

Fig. 5 There exists a continuous function $f$ taking $A$ to $a, B$ to $b, C$ to $c$ and $D$ to $d$

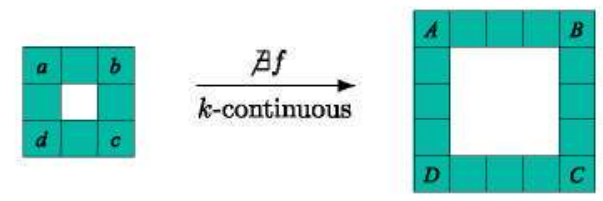

Fig. 6 There is not a continuous function $f$ taking $a$ to $A, b$ to $B, c$ to $C$ and $d$ to $D$

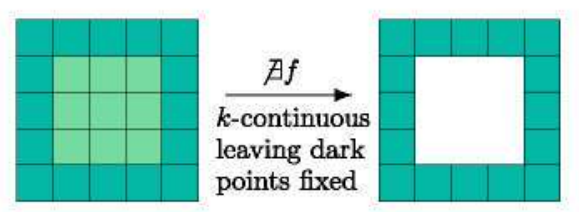

Fig. 7 The $k$-boundary of a square is not a digital $k$-retract

continuous functions, intermediate values property, almostfixed point theorem, Lipschitz conditions, one-to-oneness, etc. Boxer [1-3] expanded this notion to digital homeomorphisms, retractions, extensions, homotopies, digital fundamental group, induced homomorphisms, etc. (see also [12] and [14] for previous related results).

In particular, Boxer proved in [1] that the $k$-boundary of a digital square is not a digital $k$-retract (see Definition 9) of the square.

However, the same techniques used to prove this fact can be used to prove that neither is the outer $k$-boundary of an annulus a $k$-retract of the annulus.

Note that Fig. 7 agrees with what happens if we consider the sets as subsets of $\mathbb{R}^{2}$. However, Fig. 8 does not agree, since in $\mathbb{R}^{2}$, the outer $k$-boundary, or the outer half, of an annulus, is a digital $k$-retract of the annulus.

\section{Digitally Continuous Multivalued Functions}

As noted in the previous section, given a hollow $3 \times 3$-square $S_{3}$ and a hollow $5 \times 5$-square $S_{5}$, for $k \in\{4,8\}$, there is not a $k$-continuous function between them, which takes the corners of $S_{3}$ to the corresponding corners of the $S_{5}$.

The point here is that no matter how we define the image of the rest of the points, connectedness would not be preserved. However, we would preserve connectedness if we could define the image of each point between two corners of $S_{3}$ as the whole set of points between the corresponding corners of $S_{5}$, i.e., using multivalued functions.
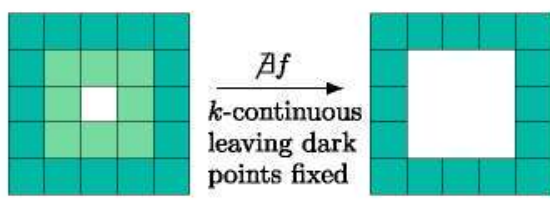

Fig. 8 The outer $k$-boundary of an annulus is not a digital $k$-retract

In this section we show how it is possible to define a notion of continuity for multivalued functions in such a way that the limitations discussed above of digitally continuous single valued functions are alleviated (see Proposition 2).

The definitions and results in this section were first introduced in [7].

Definition 6 The first subdivision of $\mathbb{Z}^{n}$ is formed by the set

$\mathbb{Z}_{1}^{n}=\left\{\left(\frac{z_{1}}{3}, \frac{z_{2}}{3}, \ldots, \frac{z_{n}}{3}\right) \mid\left(z_{1}, z_{2}, \ldots, z_{n}\right) \in \mathbb{Z}^{n}\right\}$

and the $3^{n}: 1$ function $i: \mathbb{Z}_{1}^{n} \longrightarrow \mathbb{Z}^{n}$ given by

$i\left(\frac{z_{1}}{3}, \frac{z_{2}}{3}, \ldots, \frac{z_{n}}{3}\right)=\left(z_{1}^{\prime}, z_{2}^{\prime}, \ldots, z_{n}^{\prime}\right)$

where $\left(z_{1}^{\prime}, z_{2}^{\prime}, \ldots, z_{n}^{\prime}\right)$ is the point in $\mathbb{Z}^{n}$ which is closest to $\left(\frac{z_{1}}{3}, \frac{z_{2}}{3}, \ldots, \frac{z_{n}}{3}\right)$ in the Euclidean metric.

The $r$-th subdivision of $\mathbb{Z}^{n}$ is formed by the set

$\mathbb{Z}_{r}^{n}=\left\{\left(\frac{z_{1}}{3^{r}}, \frac{z_{2}}{3^{r}}, \ldots, \frac{z_{n}}{3^{r}}\right) \mid\left(z_{1}, z_{2}, \ldots, z_{n}\right) \in \mathbb{Z}^{n}\right\}$

and the $3^{n r}: 1$ function $i_{r}: \mathbb{Z}_{r}^{n} \longrightarrow \mathbb{Z}^{n}$ given by

$i_{r}\left(\frac{z_{1}}{3^{r}}, \frac{z_{2}}{3^{r}}, \ldots, \frac{z_{n}}{3^{r}}\right)=\left(z_{1}^{\prime}, z_{2}^{\prime}, \ldots, z_{n}^{\prime}\right)$

where $\left(z_{1}^{\prime}, z_{2}^{\prime}, \ldots, z_{n}^{\prime}\right)$ is the point in $\mathbb{Z}^{n}$ which is closest to $\left(\frac{z_{1}}{3^{r}}, \frac{z_{2}}{3^{r}}, \ldots, \frac{z_{n}}{3^{r}}\right)$ in the Euclidean metric.

Moreover, if we consider in $\mathbb{Z}^{n}$ a $k$-adjacency relation, we can consider in $\mathbb{Z}_{r}^{n}$ the induced adjacency relation, i.e., $\left(\frac{z_{1}}{3^{r}}, \frac{z_{2}}{3^{r}}, \ldots, \frac{z_{n}}{3^{r}}\right)$ and $\left(\frac{z_{1}^{\prime}}{3^{r}}, \frac{z_{2}^{\prime}}{3^{r}}, \ldots, \frac{z_{n}^{\prime}}{3^{r}}\right)$ are $k$-adjacent if and only if $\left(z_{1}, z_{2}, \ldots, z_{n}\right)$ is $k$-adjacent to $\left(z_{1}^{\prime}, z_{2}^{\prime}, \ldots, z_{n}^{\prime}\right)$.

Proposition $1 i_{r}$ is $k$-continuous as a function between digital spaces.

Definition 7 Given $X \subset \mathbb{Z}^{n}$, the $r$-th subdivision of $X$ is the set $X_{r}=i_{r}^{-1}(X)$.

Intuitively, if we consider $X$ made of pixels (respectively, voxels), the $r$ th subdivision of $X$ consists in replacing each pixel with $9^{r}$ pixels (respectively, $27^{r}$ voxels) and the function $i_{r}$ is like an inclusion in the geometric sense (see Fig. 9).

Remark 1 Given $X, Y \subset \mathbb{Z}^{n}$, any function $f: X_{r} \longrightarrow Y$ induces in an immediate way a multivalued function $F$ : $X \longrightarrow Y$ where $F(x)=\bigcup_{x^{\prime} \in i_{r}^{-1}(x)} f\left(x^{\prime}\right)$. 


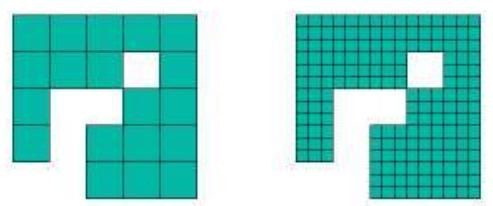

Fig. 9 A digital set (left) and its first subdivision (right)

Definition 8 Consider $X, Y \subset \mathbb{Z}^{n}$. A multivalued function $F: X \longrightarrow Y$ is said to be a $\left(k, k^{\prime}\right)$-continuous multivalued function if it is induced by a $\left(k, k^{\prime}\right)$-continuous (singlevalued) function from $X_{r}$ to $Y$ for some $r \in \mathbb{N}$.

In the following remark we state some properties of digitally continuous multivalued functions proved in [7]. For more results and details the reader is referred to [7].

Remark 2 Any single-valued digitally continuous function is continuous as a multivalued function. In particular, any constant function is continuous as a multivalued function. Moreover, if $F: X \longrightarrow Y\left(X, Y \subset \mathbb{Z}^{2}\right)$ is a $\left(k, k^{\prime}\right)$ continuous multivalued function, then:

(i) $F(x)$ is $k^{\prime}$-connected, for every $x \in X$.

(ii) If $x$ and $y$ are $k$-adjacent points of $X$, then $F(x) \cup F(y)$ is a $k^{\prime}$-connected subset of $Y$.

(iii) $F$ takes $k$-connected sets to $k^{\prime}$-connected sets.

(iv) If $X^{\prime} \subset X$ then $\left.F\right|_{X^{\prime}}: X^{\prime} \longrightarrow Y$ is a $\left(k, k^{\prime}\right)$-continuous multivalued function.

Moreover, the composition of continuous multivalued functions is a continuous multivalued function.

Definition 9 Let $X \subset \mathbb{Z}^{2}$ and $Y \subset X$. We say that $Y$ is a $k$-retract of $X$ if there exists a $k$-continuous multivalued function $F: X \longrightarrow Y$ (a multivalued $k$-retraction) such that $F(y)=\{y\}$ if $y \in Y$.

If moreover $F(x) \subset \mathcal{N}(x)$ for every $x \in X \backslash Y$, we say that $F$ is a multivalued $(\mathcal{N}, k)$-retraction.

The following results are given in [7].

\section{Proposition 2 The following holds:}

(i) The $k$-boundary $\partial_{k} X$ of a square $X$, with nonempty interior, is not a $k$-retract of $X$.

(ii) The outer $k$-boundary $\partial_{k} X$ of an annulus $X$ is a $k$ retract of $X$.

Result (ii) solves one of the problems presented when using single-valued functions (see Fig. 8).

In the rest of the paper we show how this notion of digital continuity also allows us to characterize basic morphological operations as dilation and closing, or thinning algorithms.
Fig. 10 Labels of $\mathcal{N}(p)$ and

$i^{-1}(p)$

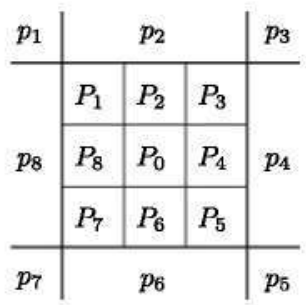

\section{Morphological Operators as Digitally Continuous Multivalued Functions}

In this section we consider the basic operations in mathematical morphology: dilation, erosion, closing, and opening operators (see [22] for their definitions). We will denote by $D_{k}(X), E_{k}(X), C_{k}(X), O_{k}(X)$, respectively, the dilation, erosion, closing, and opening of a digital set $X$ in $\mathbb{Z}^{2}$, using as a structuring element the set formed by a point and its $k$-neighbors.

We will show that these operators can be modeled as digitally continuous multivalued functions, which we will denote, respectively, by $D_{k}, E_{k}, C_{k}, O_{k}$. Note that, then, the notation $D_{k}(X)$ will not only indicate the image of $X$ under the function $D_{k}$, but also the set that is the $k$-dilation of $X$. Note, however, that these two sets agree. The same can be said for the other operators.

We start the section with a theorem which shows how the dilation operator can be modeled by a continuous multivalued function.

Theorem 1 Given $X \subset \mathbb{Z}^{2}$, the multivalued functions $D_{k}$ : $X \longrightarrow D_{k}(X)$ given by $D_{k}(x)=\mathcal{N}_{k}(x) \cup\{x\}, k=4,8$, are digitally $k$ and $\vec{k}$-continuous.

Proof Consider the first subdivision of $X$ and the points of $i^{-1}(p)$ and those in $\mathcal{N}(p)$ labeled as in Fig. 10.

Then $D_{8}$ is induced by the function $d_{8}$ given by $d_{8}\left(P_{i}\right)=$ $p_{i}$ if $i \neq 0$, and $d_{8}\left(P_{0}\right)=p$, while $D_{4}$ is induced by the function $d_{4}$ given by $d_{4}\left(P_{i}\right)=p_{i}$ if $i \in\{2,4,6,8\}$ and $d_{4}\left(P_{i}\right)=p$ if $i \in\{0,1,3,5,7\}$. It is immediate to check that $D_{k}$ is $k$ and $\bar{k}$-continuous.

The erosion operation cannot be adequately modeled as a digitally continuous multivalued function on the set of black pixels since it can transform a connected set into a disconnected set, or even delete it (for example, the erosion of a curve is the empty set and, in general, the erosion of two discs connected by a curve would be the disconnected union of two smaller discs). However, since the erosion of a set agrees with the dilation of its complement, the erosion operator can be modeled by a continuous multivalued function on the set of white pixels. 
Corollary 1 Given $X \subset \mathbb{Z}^{2}$, the multivalued function $E_{k}$ : $\mathbb{Z}^{2} \backslash X \longrightarrow \mathbb{Z}^{2}$, given by $E_{k}(x)=\mathcal{N}_{k}(x) \cup\{x\}$ is digitally $k$ and $\bar{k}$-continious.

Although the erosion operation cannot be modeled as a digitally continuous inultivalued function on the set of black pixels, when combined with the dilation we obtain the closing operation which can be modeled a digitally continuous multivalued function. Recall, as noted at the beginning of the section, that $C_{k}(X)$ will not only indicate the image of $X$ under the multivalued function $C_{k}$, but also the set that is the $k$-closing (a $k$-dilation composed with a $k$-erosion) of $X$.

Theorem 2 Given $X \subset \mathbb{Z}^{2}$, the multivalued function $C_{k}$ : $X \longrightarrow C_{k}(X)$, given by $C_{k}(x)=\{x\}$ for every $x \in X \backslash \partial_{k} X$ and $C_{k}(x)=\left(\{x\} \cup \mathcal{N}_{k}(x)\right) \cap C_{k}(X)$ for every $x \in \partial_{k} X$, is digitally $k$-continuous.

Proof With the notation of Theorem $1, C_{k}$ is induced by $c_{k}: i^{-1}(X) \longrightarrow C_{k}(X)$ given by

$c_{k}\left(P_{i}\right)= \begin{cases}p_{i} & \text { if } i \neq 0 \text { and } p_{i} \in C_{k}(X) . \\ \rho & \text { if } i=0 \text { or } i \neq 0 \text { and } p_{i} \notin C_{k}(X) .\end{cases}$

It is immediate to check that $C_{k}$ is $k$-continuous.

As it happens in the case of the erosion, the opening operation (erosion composed with dilation) cannot be adequately modeled as a digitally continuous multivalued function on the set of black pixels (the same examples used for the erosion also work for the opening). However, since the opening of a set agrees with the closing of its complement [22], the $k$-opening operator can be modeled by a $k$-continuous multivalued function on the set of white pixels.

Corollary 2 Given $X \subset \mathbb{Z}^{2}$, the $k$-opening ( $k$-erosion $+k$ dilation) operation on $X$ can be modeled as a digitally $k$ continwus multivalued function $O_{k}: \mathbb{Z}^{2} \backslash X \longrightarrow \mathbb{Z}^{2}$ on the set of white pixels.

Theorems 1 and 2 are particular cases of the following result:

Theorem 3 Given $X \subset \mathbb{Z}^{2}$, every multivalued finction $F$ : $X \longrightarrow \mathbb{Z}^{2}$ such that $x \in F(x) \subset \mathcal{N}_{k}(x) \cup\{x\}$ for every $x \in$ $X$, is a digitally $k$-continuous multivalued function.

Proof Consider the first subdivision of $X$ and the points of $i^{-1}(p)$ and those in $\mathcal{N}(p)$ labeled as in Fig. 10. Then $F$ is induced by $f: i^{-1}(X) \longrightarrow F(X)$ given by

$f\left(P_{i}\right)= \begin{cases}p_{i} & \text { if } i \neq 0 \text { and } p_{i} \in F(x), \\ p & \text { if } i=0 \text { or } i \neq 0 \text { and } p_{i} \notin F(x) .\end{cases}$

which is a continuous function.
Corollary 3 Given $X \subset \mathbb{Z}^{2}$ and $p \in \mathbb{Z}^{2} k$-adjacent to $X$, then $F: X \longrightarrow X \cup\{p\}$ given $b y$

$F(x)= \begin{cases}x & \text { if } x \text { is not } k \text {-adjacent to } p \\ \{x, p\} & \text { if } x \text { is } k \text {-adjacent to } p\end{cases}$

is a digitally $k$-continuous multivalued function.

Corollary 4 Given $X \subset \mathbb{Z}^{2}$ and a structuring element $B$ (see [22]) containing the origin and contained in $a 3 \times 3$ square, then the dilation of $X$ by $B$ can be modeled as a digitally 8-continuous multivalued function.

Corollary 5 Given $X \subset \mathbb{Z}^{2}$ and given $Y \subset \mathbb{Z}^{2}, k$-connected and $k$-adjacent to $X$, there exists a surjective digitally continuous multivalued function $F: X \longrightarrow X \cup Y$ such that $F(x)=\{x\}$ if $x$ is not $k$-adjacent to $Y$.

Proof Since $Y$ is $k$-connected and $k$-adjacent to $X$, there exists an ordering $p_{1}, p_{2}, \ldots p_{n}$ of the points of $Y$ (where the $p_{i}$ need not be pairwise distinct) such that $p_{1}$ is $k$ adjacent to $X$ and $p_{i}$ is $k$-adjacent to (or contained in) $X \cup\left\{p_{1}, p_{2}, \ldots, p_{i-1}\right\}$, for every $i \in\{2,3, \ldots, n\}$.

Consider $F_{1}: X \longrightarrow X \cup\left\{p_{1}\right\}$ given by

$F_{1}(x)= \begin{cases}x & \text { if } x \text { is not } k \text {-adjacent to } p_{1} \\ \left\{x, p_{1}\right\} & \text { if } x \text { is } k \text {-adjacent to } p_{1}\end{cases}$

which is a digitally continuous multivalued function. For every $i \in\{2,3, \ldots, n\}$ such that $p_{i}$ is $k$-adjacent to $X \cup$ $\left\{p_{1}, p_{2}, \ldots, p_{i-1}\right\}$, consider

$F_{i}: X \cup\left\{p_{1}, p_{2}, \ldots, p_{i-1}\right\} \longrightarrow X \cup\left\{p_{1}, p_{2}, \ldots, p_{i}\right\}$

given by

$F_{i}(x)= \begin{cases}x & \text { if } x \text { is not } k \text {-adjacent to } p_{i}, \\ \left\{x, p_{i}\right\} & \text { if } x \text { is } k \text {-adjacent to } p_{i}\end{cases}$

which is also is a digitally continuous multivalued function, while, if $p_{i}$ is contained in $X \cup\left\{p_{1}, p_{2}, \ldots, p_{i-1}\right\}$, we consider

$F_{i}: X \cup\left\{p_{1}, p_{2}, \ldots, p_{i-1}\right\} \rightarrow X \cup\left\{p_{1}, p_{2}, \ldots, p_{i}\right\}$,

such that $F_{i}$ is the identity.

Then, since the composition of digitally continuous multivalued functions is a digitally continuous multivalued function (see Remark 2), the multivalued function $F=F_{n}$ 。 $F_{n-1} \circ \cdots \circ F_{2} \circ F_{1}$ satisfies the conclusion of the corollary.

We end this section with a result which, although a consequence of the previous result, is better stated and proved in 
terms of notions and properties of mathematical morphology. It is based on the fact that a morphological operation with a large strncturing element can be decomposed into a sequence of operations with smaller strncturing elements [22], and, on the other hand, that the composition of digitally continuons multivalued fnnctions is a digitally continnons multivalued function.

Corollary 6 Given $X \subset \mathbb{Z}^{2}$, and a rectangular structuring element $B$ centered at the origin and containing it, then the dilation of $X$ by $B$ can be modeled as digitally continious multivalued function.

Proof A dilation with a rectangular structnring element of sides $2 m+1$ and $2 n+1$ pixels is equivalent to a dilation with a horizontal line of $2 m+1$ pixels followed by a dilation with a vertical line of $2 n+1$ pixels. On the ohther hand, a dilation with a horizontal (respectively, vertical line) of $2 m+1$ (respectively, $2 n+1$ ) pixels is equivalent to a seqnence of $m$ (respectively, $n$ ) dilations with a horizontal (respectively, vertical) line of 3 pixels. (See, for example, [22, p, 640]).

In the particular case of a dilation by a square of width $2 n+1$ pixels, this can be more easily done with $n$ successive dilations with a $3 \times 3$ square.

The result follows from the fact that the composition of digitally continuous multivalued functions is a digitally continuous multivalued function.

\section{Continuous Multivalued Functions and Deletion of Simple Points}

It may seem that the family of continuous multivalued functions could be too broad, therefore not having good properties. In this section we show that this is not the case. We show, in particular, that the existence of a $k$-continuous multivalued function from a set $X$ to $X \backslash\{\rho\}$ which leaves invariant $X \backslash\{\rho\}$ is closely related to $p$ being a $k$-simple point of $X$.

Let $X \subset \mathbb{Z}^{2}$ and $D \subset X . D$ is called $k$-deletable $(k=$ 4,8 ) in $X$ if its deletion does not change the topology of $X$ in the sense that after deleting $D$ :

- no $k$-connected component of $X$ vanishes or is split into multiple components,

- no $\bar{k}$-connected hole in $X$ is created or is merged with the background or with another such hole.

Here, as noted in Sect. $1, \bar{k}=4$ if $k=8$ and $\bar{k}=8$ if $k=4$.

Let $X \subset \mathbb{Z}^{2}$. A point $p \in X$ is called $k$-simple $(k=4,8)$ in $X$ (see [15]) if $\{p\}$ is $k$-deletable in $X$.

A $k$-simple point can be locally detected by the following characterization [20]:
Theorem 4 Let $X \subset \mathbb{Z}^{2}$. A point $p \in X$ is $k$-simple if it is a $k$-boundary point of $X$ and the number of $k$-connected components of $\mathcal{N}(p) \cap X$ which are $k$-adjacent to $p$ is equal to 1.

The following theorem was proved in $[7,8]$ where two different algorithms were given. The differences in the algorithms in these papers are basically two: in the second one we only require consideration of the first subdivision of $X$ (and not the second as happened in the first one), and we obtain smaller inages for the deleted simple points. Althongh we state the resnlt for $k$-connected sets, this is not a loss of generality because for a general set $X$ it wonld be applied to the connected component containing the simple point we want to delete.

Theorem 5 Let $X \subset \mathbb{Z}^{2}$ be $k$-connected and consider $p \in X$. Suppose that there exists a $k$-continuous multivalued function $F: X \longrightarrow X \backslash\{p\}$ such that $F(x)=\{x\}$ if $x \neq p$ and $F(p) \subset \mathcal{N}(p)$. Then $p$ is a $k$-simple point.

The converse is true ander the following conditions:

(a) for $k=8$ it is always true and, moreover, we can impose that $F(p) \subset \mathcal{N}_{4}(p)$ whenever $p$ is not 4-isolated,

(b) for $k=4$ it is trice if and only if $p$ is not 8-interior to $X$.

Proof The proof of this result can be found in [8]. However, since it rests on an argument that we will use throughout the paper, we reproduce here the proof of the direct implication.

Suppose that $F$ is induced by $f_{r}: X_{r} \longrightarrow X$. Then $f_{r}(x)=i_{r}(x)$ for every $x \in X_{r}$ such that $i_{r}(x) \neq p$.

Suppose that $p$ is not $k$-simple. We have two possibilities: $p$ is a $k$-boundary point with at least two different $k$ connected components of $\mathcal{N}(p) \cap X$ which are $k$-adjacent to $p$, or $p$ is an interior point.

In the first case, let $A$ and $B$ be any two such components. Consider any $x_{r} \in i_{r}^{-1}(p) k$-adjacent to $i_{r}^{-1}(A)$. Then $x=f_{r}\left(x_{r}\right)$ must be $k$-adjacent to $A$ or contained in $A$ (since $F(A)=A$ ), and since $A$ is a $k$-connected component of $\mathcal{N}(p) \cap X$, we have $f_{r}\left(x_{r}\right) \in A$. On the other hand, there also exists $y_{r} \in i_{r}^{-1}(p) k$-adjacent to $i_{r}^{-1}(B)$ and, hence, $f_{r}\left(y_{r}\right)=y \in B$. Consider

$\left\{z_{0}=x_{r}, z_{1}, z_{2} m, \ldots, z_{m-1}, z_{m}=y_{r}\right\} \subset i_{r}^{-1}(p)$

such that, for every $i=1,2, \ldots, m, z_{i}$ is $k$-adjacent to $z_{i-1}$. Then

$\left\{f_{r}\left(z_{0}\right)=x, f_{r}\left(z_{1}\right), f_{r}\left(z_{2}\right), \ldots, f_{r}\left(z_{m-1}\right), f_{r}\left(z_{m}\right)=y\right\}$

is a $k$-path in $\mathcal{N}(p) \cap X$ from $x$ to $y$. Contradiction.

For the case of $p$ interior and the converse implication we refer the reader to [8], although, as an illustration, we show how to construct $F$ in two particular cases, giving alternative constructions to those in $[7,8]$. 

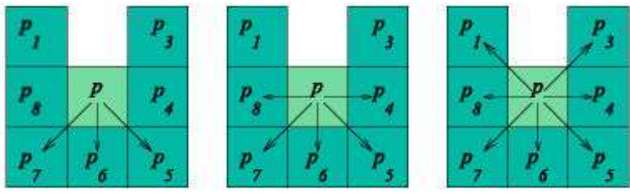

Fig. $11 F_{1}($ left $), F_{2}$ (center) and $F_{3}($ right $)$ multivalued 4-continuous functions deleting a 4 -simple point

Fig. 12 The 4-continuous single valued function $f_{1}$ inducing $F_{1}$
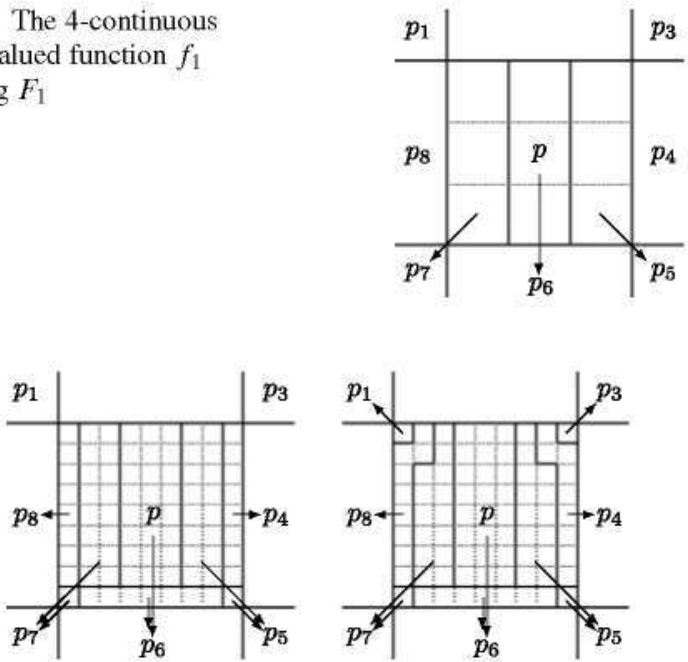

Fig. 13 Left: A single valued function $f_{2}$ inducing $F_{2}$. Right: A single valued function $f_{3}$ inducing $F_{3}$

If $p \in X$ is a 4-simple point such that $\mathcal{N}(p) \cap X=$ $\left\{p_{1}, p_{3}, p_{4}, p_{5}, p_{6}, p_{7}, p_{8}\right\}$, we show in Fig. 11 three different 4-continuous multivalued functions such that $F(x)=x$ if $x \neq p$ (the image of $p$ is defined by the arrows).

The function $F_{1}$ on the left has the property that the image of $p$ has the smallest possible number of points. The function $F_{3}$ on the right, on the other hand, has the largest possible size. In general, it is possible to prove, in a similar way as in $[7,8]$, that the function which assigns to a $k$-simple point $p$ the whole (and unique) $k$-connected components of $\mathcal{N}(p) \cap X k$-adjacent to $p$ is $k$-continuous (as it happens with $F_{3}$ ). On the other hand, it is possible to construct an algorithm to obtain the image of $p$ with the smallest possible number of points (like $F_{1}$ ).

To see why $F_{1}$ is 4-continuous we consider the first subdivision $X_{1}$ of $X$ and $f_{1}: X_{1} \longrightarrow X$ such that $f_{1}\left(x^{\prime}\right)=x$ for every $x^{\prime} \in i_{1}^{-1}(x), x \neq p$, and such that $f_{1}$ is defined in $i_{1}^{-1}(p)$ as shown by the arrows in Fig. 12.

For the functions $F_{2}$ and $F_{3}$ we have to consider the second subdivision $X_{2}$ of $X$, divide $i_{2}^{-1}(p)$ in groups and define $f_{2}$ and $f_{3}$ in the points of $i_{2}^{-1}(p)$ as shown by the arrows in Fig. 13.

If we consider now $p$ as an 8 -simple point, then we show in Fig. 14 three different 8 -continuous multivalued functions such that $F(x)=x$ if $x \neq p$.
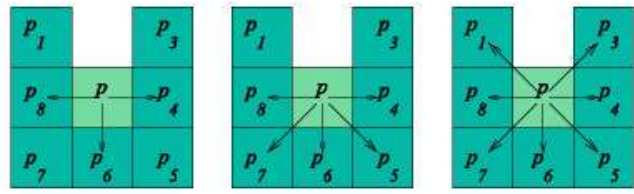

Fig. $14 F_{1}$ (left), $F_{2}$ (center) and $F_{3}($ right $)$ multivalued 8-continuous functions deleting an 8 -simple point

Fig. 15 An 8-continuous single valued function $f_{1}$ inducing $F_{1}$

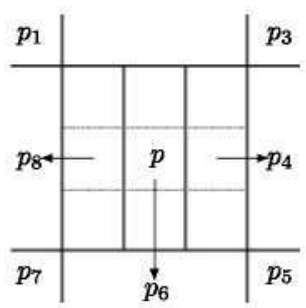

The function $F_{1}$ has the property that the image of $p$ has the smallest possible number of points, while the function $F_{3}$ on the right has the largest possible size.

To see why $F_{1}$ is 8 -continuous we consider the first subdivision $X_{1}$ of $X$ and we divide $i_{1}^{-1}(p)$ in groups and define $f_{1}$ as shown by the arrows in Fig. 15 .

For the two other functions $F_{2}$ and $F_{3}$, the same groups of Fig. 13, for the case $k=4$, are still valid. This ends the proof of the theorem.

Since the composition of $k$-continuous multivalued functions is a $k$-continuous multivalued function, we have the following result [7]:

Corollary 7 Let $X \subset \mathbb{Z}^{2}$ be $k$-connected, $D=\left\{p_{i}\right\}_{i=1}^{n}$, $Y=X \backslash D$, such that $p_{1}$ is $k$-simple in $X$ and is not an 8-interior point of $X$, and for $2 \leq i \leq n$ we have $p_{i}$ is $k$ simple in $X \backslash \bigcup_{j=1}^{i-1}\left\{p_{j}\right\}$ and is not an 8-interior point of $X \backslash \bigcup_{j=1}^{i-1}\left\{p_{j}\right\}$. Then there exists a $k$-continuous multivalued $k$-retraction from $X$ to $Y$.

Remark 3 The converse is not true since any constant function is continuous but not all sets (for example, digital simple closed curves like the ones in Fig. 5) can be reduced by a sequential deletion of simple points to a single point. However, in Sect. 7 we give a partial converse result using the notion of $(\mathcal{N}, k)$-retraction.

Remark 4 It is easy to see that if $\mathcal{N}(p) \cap X$ is as in Fig. 11, although $p$ is 4 -simple and 8 -simple, any single-valued function $f: X \longrightarrow X \backslash\{p\}$, such that $f(x)=x$ if $x \neq p$, cannot be neither 4-continuous nor 8-continuous, hence an analog of Theorem 5 does not hold for Rosenfeld's digitally continuous single-valued functions. 
Fig. 16 A non-deletable pair of $k$-simple points $(k=4,8)$

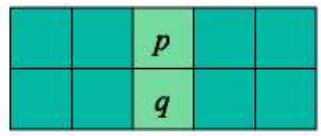

\section{Continuous Multivalued Functions and Deletion of Simple Pairs}

It is interesting to note that the ideas in Theorem 5 can be also applied to pairs of 4-adjacent points whose simultaneous deletion does not change the $k$-topology. Such points, called $k$-simple pairs, are essential to verify the correctedness of parallel thinning algorithms and are locally characterized as follows:

A pair $\{p, q\}$ of 4-adjacent points of $X$ is a $k$-simple pair if and only if at least one of them is not a $k$-interior point and the number of $k$-components of $\mathcal{N}(p, q) \cap X$ that are $k$ adjacent to $\{p, q\}$ is 1 , where $\mathcal{N}(p, q)=(\mathcal{N}(p) \cup \mathcal{N}(q)) \backslash$ $\{p, q\}$.

It is well known that a set formed by two 4-adjacent $k$ simple points does not need to be a $k$-simple pair (e.g., see Fig. 16).

On the other hand, a $k$-simple pair does not need to be composed of $k$-simple points. However, if $\{p, q\}$ is a $k$ simple pair, then we can order them in such a way that one of them, say $p$, is a $k$-simple point in $X$, and $q$ is a $k$-simple point in $X \backslash\{p\}$. This result, that we will use later, is a consequence of the following more general result [18]:

Theorem 6 ([18]) The deletion of a subset $D$ of $X$ does not change the k-topology of $X$ if and only if there is an ordering of the points of $D, D=\left\{p_{i}\right\}_{i=1}^{n}$, such that $p_{1}$ is a $k$-simple point of $X$ and, for $2 \leq i \leq n, p_{i}$ is a $k$-simple point of $X \backslash \bigcup_{j=1}^{i-1}\left\{p_{j}\right\}$.

In the following theorem we show that the deletion of a $k$-simple pair can be modeled as a digitally $k$-continuous multivalued function.

Theorem 7 Let $X \subset \mathbb{Z}^{2}$ be $k$-connected and consider a pair $\{p, q\} \subset X$ of 4-adjacent points of $X$. Suppose that there exists a $k$-continuous multivalued function $F: X \rightarrow X \backslash$ $\{p, q\}$ such that $F(x)=\{x\}$ if $x \notin\{p, q\}, F(p) \cup F(q) \subset$ $\mathcal{N}(p, q)$. Then $\{p, q\}$ is a $k$-simple pair.

The converse is true under the following conditions:

(a) for $k=8$ it is always true and, moreover, we can impose that $F(\{p, q\}) \subset \mathcal{N}_{4}(p, q)=\left(\mathcal{N}_{4}(p) \cup \mathcal{N}_{4}(q)\right) \backslash\{p, q\}$ whenever $\{p, q\}$ is not a 4-connected component of $X$,

(b) for $k=4$ it is true if and only if $p$ or $q$ is not 8-interior to $X$.

Proof To prove the first assertion, suppose that $F$ is induced by $f_{r}: X_{r} \longrightarrow X$. Then $f_{r}(x)=i_{r}(x)$ for every $x \in X_{r}$ such that $i_{r}(x) \notin\{p, q\}$.
Fig. 17 Labels of the points in $\mathcal{N}(p, q)$

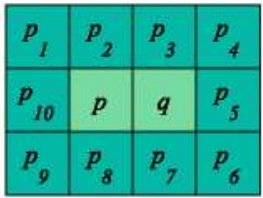

Suppose that $\{p, q\}$ is not a $k$-simple pair. Then we have two possibilities: there are at least two different $k$-connected components of $\mathcal{N}(p, q) \cap X$ which are $k$-adjacent to $\{p, q\}$, or $p$ and $q$ are both $k$-interior points.

In the first case, let $A$ and $B$ be any two such components. Consider any $x_{r} \in i_{r}^{-1}(\{p, q\}) k$-adjacent to $i_{r}^{-1}(A)$. Then $x=f_{r}\left(x_{r}\right)$ must be $k$-adjacent to $A$ or contained in $A$ (since $F(A)=A$ ), and since $A$ is a $k$-connected component of $\mathcal{N}(p) \cap X$, then $f_{r}\left(x_{r}\right) \in A$. Similarly, there exists $y_{r} \in i_{r}^{-1}(\{p, q\}) k$-adjacent to $i_{r}^{-1}(B)$ and, hence, $f_{r}\left(y_{r}\right)=$ $y \in B$. Consider

$z_{0}=x_{r}, z_{1}, z_{2}, \ldots, z_{m-1}, z_{m}=y_{r} \subset i_{r}^{-1}(\{p, q\})$

such that $z_{i}$ is $k$-adjacent to $z_{i-1}$ for $i=1,2, \ldots, m$. Then

$\left\{f_{r}\left(z_{0}\right)=x, f_{r}\left(z_{1}\right), \ldots, f_{r}\left(z_{m}\right)=y\right\} \subset \mathcal{N}(p, q) \cap X$

is a $k$-path in $\mathcal{N}(p, q) \cap X$ from $x$ to $y$. Contradiction.

Suppose now that $p$ and $q$ are $k$ interior points. Denote the points in $\mathcal{N}(p, q) \subset X$ as in Fig. 17.

Define $G: \mathcal{N}(p) \cup\{p\} \longrightarrow \mathcal{N}(p, q) \cup\{p, q\}$ and $H:$ $\mathcal{N}(p, q) \cup\{p, q\} \longrightarrow \mathcal{N}(p) \cup\{p\}, 8$ and 4-continuous multivalued functions given by $G(p)=\{p, q\}, H(\{p, q\})=p$ and the following tables

\begin{tabular}{lllllllll}
\hline$x$ & $p_{1}$ & $p_{10}$ & $p_{9}$ & $p_{2}$ & $p_{8}$ & $p_{3}$ & $q$ & $p_{7}$ \\
$G(x)$ & $p_{1}$ & $p_{10}$ & $p_{9}$ & $\left\{p_{2}, p_{3}\right\}$ & $\left\{p_{7}, p_{8}\right\}$ & $p_{4}$ & $p_{5}$ & $p_{6}$ \\
\hline
\end{tabular}

$\begin{array}{lllllllll}x & p_{1} & p_{10} & p_{9} & \left\{p_{2}, p_{3}\right\} & \left\{p_{7}, p_{8}\right\} & p_{4} & p_{5} & p_{6}\end{array}$

\begin{tabular}{lllllllll}
$H(x)$ & $p_{1}$ & $p_{10}$ & $p_{9}$ & $p_{2}$ & $p_{8}$ & $p_{3}$ & $q$ & $p_{7}$ \\
\hline
\end{tabular}

i.e. $G$ (respectively, $H$ ) takes the left vertical side of $\mathcal{N}(p) \cup$ $\{p\}$ (respectively, $\mathcal{N}(p, q) \cup\{p, q\}$ ) to the left vertical side of $\mathcal{N}(p, q) \cup\{p, q\}$ (respectively, $\mathcal{N}(p) \cup\{p\}$ ), the right vertical side to the right vertical side, and the middle vertical part to the middle vertical part, the latter in a $1: 2$ (respectively, $2: 1)$ way.

Then $H F G: \mathcal{N}(p) \cup\{p\} \rightarrow \mathcal{N}(p) \cup\{p\}$ is a $k$-continuous multivalued function such that $\operatorname{HFG}(x)=\{x\}$ if $x \neq p$ and $H F G(p) \subset \mathcal{N}(p)$. Therefore, by Theorem 5, $p$ would be a $k$-simple point and this is a contradiction because $p$ is a $k$-interior point.

We prove now the converse statement.

Suppose first that $k=8$. If $\{p, q\}$ is an 8 -simple pair, then, by Theorem 6 , we can order them in such a way that 
Fig. 18 A 4-simple pair of 8 -interior points

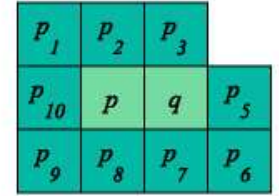

one of them, say $p$, is an 8-simple point, and $q$ is an 8simple point in $X \backslash\{p\}$. Since $p$ is 8 -simple, by Theorem 5 , there exists an 8-continuous multivalued function $F_{1}$ : $X \longrightarrow X \backslash\{p\}$ such that $F_{1}(x)=\{x\}$ if $x \neq p$ and $F_{1}(p) \subset$ $\mathcal{N}_{4}(p)$. Since $q$ is 8-simple in $X \backslash\{p\}$, then there exists an 8continuous multivalued function $F_{2}: X \backslash\{p\} \longrightarrow X \backslash\{p, q\}$ such that $F_{2}(x)=\{x\}$ if $x \neq q$ and $F_{2}(q) \subset \mathcal{N}_{4}(q)$. Then the composition $F=F_{2} F_{1}$ satisfies the statement of the theorem.

Suppose now that $k=4$. If $\{p, q\}$ is a 4 -simple pair we can distinguish three cases:

(a) Neither $p$ nor $q$ is 8 -interior. In this case the proof is similar to that for $k=8$.

(b) Just one of them, say $p$, is 8 -interior. Then $q$ must be 8 -simple and the proof above is again valid.

(c) Both $p$ and $q$ are 8 -interior. Then (see Fig. 18) $\left\{p_{2}, p_{3}\right.$, $\left.p_{5}, p_{7}, p_{8}, p_{10}\right\} \subset X$. But, since $\{p, q\}$ is a 4-simple pair, there is only one 4-connected component of $\mathcal{N}(p, q) \cap X$ 4-adjacent to $\{p, q\}$. This implies that $\mathcal{N}(p, q) \cap X$ must be as in Fig. 18 (or a rotation or a symmetry of it).

Suppose that there exists a 4-continuous multivalued function $F: X \longrightarrow X \backslash\{p, q\}$ such that $F(x)=\{x\}$ if $x \notin\{p, q\}$ and $F(p) \cup F(q) \subset \mathcal{N}(p, q) \cap X$, and suppose that $F$ is induced by $f_{r}: X_{r} \longrightarrow X$. Then, since $f_{r}(x)=p_{i}$ for every $x \in X_{r}$ such that $i_{r}(x)=p_{i}$, if we consider the upper rightmost point $x \in i_{r}^{-1}(q)$, it is not possible to define $f_{r}(x)$ in such a way that $f_{r}$ is 4continuous.

This ends the proof of the theorem.

Observe that in the theorem above we do not require that $F(p) \subset \mathcal{N}(p)$ or $F(q) \subset \mathcal{N}(q)$, as in $(\mathcal{N}, k)$-retractions. If we consider this additional requirement we obtain the following result:

Theorem 8 Let $X \subset \mathbb{Z}^{2}$ be $k$-connected and consider a pair $\{p, q\} \subset X$ of 4-adjacent points of $X$. Suppose that there exists a $k$-continuous multivalued function $F: X \longrightarrow X \backslash$ $\{p, q\}$ such that $F(x)=\{x\}$ if $x \notin\{p, q\}, F(p) \subset \mathcal{N}(p)$, $F(q) \subset \mathcal{N}(q)$ and $F(p)$ (respectively, $F(q)) k$-adjacent to $p$ (respectively, $q$ ). Then $\{p, q\}$ is a $k$-simple pair and both $p$ and $q$ are $k$-simple points.

The converse is true if and only if neither $p$ nor $q$ is 8interior to $X$.

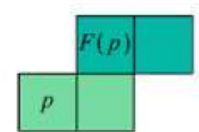

Fig. 19 There exists $(\mathcal{N}, 4)$-retraction but there is not a 4-connected component of $\mathcal{N}(p) \cap(X \backslash D)$ 4-adjacent to $p$

Before proving Theorem 8 we prove a Lemma which will be used in its proof and that of Theorem 9. Its proof is very similar to that of the direct implication of Theorem 5 . When applying it to prove Theorem 8 note that the function $F$ in the theorem is an $(\mathcal{N}, k)$-retraction $F: X \longrightarrow X \backslash\{p, q\}$.

Lemma 1 Consider $X \subset \mathbb{Z}^{2} k$-connected and $D \subset X$ such that there exists an $(\mathcal{N}, k)$-retraction $F: X \longrightarrow X \backslash D$. Then, for any $p \in D$, there is at most one $k$-connected component of $\mathcal{N}(p) \cap(X \backslash D) k$-adjacent to $p$ and, moreover, if there is one, it must contain $F(p)$.

Proof Suppose that $F$ is induced by $f_{r}: X_{r} \longrightarrow X \backslash D$. Then $f_{r}(x)=i_{r}(x)$ for every $x \in X_{r}$ such that $i_{r}(x) \in$ $X \backslash D$.

Suppose that there are two different $k$-connected components $A$ and $B$ of $\mathcal{N}(p) \cap(X \backslash D)$ which are $k$-adjacent to $p$. Consider any $x_{r} \in i_{r}^{-1}(p) k$-adjacent to $i_{r}^{-1}(A)$. Then $x=f_{r}\left(x_{r}\right)$ must be $k$-adjacent to $A$ or contained in $A$ (since $F(A)=A$ ), and since $A$ is a $k$-connected component of $\mathcal{N}(p) \cap X$, then $f_{r}\left(x_{r}\right) \in A$. Similarly, there also exists $y_{r} \in i_{r}^{-1}(p) k$-adjacent to $i_{r}^{-1}(B)$ with $f_{r}\left(y_{r}\right) \in B$. But then $F(p) \subset \mathcal{N}(p)$ is a $k$-connected set which would connect $A$ and $B$ in $\mathcal{N}(p) \cap(X \backslash D)$. Contradiction. Therefore, there exists at most one $k$-connected component of $\mathcal{N}(p) \cap(X \backslash D) k$-adjacent to $p$.

We show finally that, if there is a $k$-connected component of $\mathcal{N}(p) \cap(X \backslash D) k$-adjacent to $p$ then it must contain $F(p)$. Let $A$ be such a $k$-connected component of $\mathcal{N}(p) \cap(X \backslash D)$. Then $F(p) \subset X \backslash D$ and $F(A)=A$ must be $k$ adjacent and, hence, $F(p) \subset A$.

The example of Fig. 19 shows that the existence of an $(\mathcal{N}, k)$-retraction $F: X \longrightarrow X \backslash D$ does not guarantee, for $k=4$, the existence of a $k$-connected component of $\mathcal{N}(p) \cap$ $(X \backslash D) k$-adjacent to $p$.

Lemma 1 will be used in several parts of the paper in different situations in which we have an $(\mathcal{N}, k)$-retraction $F: X \rightarrow X \backslash D$, because this imposes restrictions on the neighborhood of a point in $D$. Some of those situations are shown in the following corollary.

Corollary 8 Consider $X \subset \mathbb{Z}^{2} k$-connected and $D \subset X$ such that there exists an $(\mathcal{N}, k)$-retraction $F: X \rightarrow X \backslash D$. Consider $p \in D$. Then:

(i) All points of $X \backslash D k$-adjacent to $p$ must be $k$-connected in $\mathcal{N}(p) \cap X$ to those of $F(p)$. 
(ii) If $p \in D \cap \partial_{k} X$ is not $k$-simple, there exists $d \in D k$ adjacent to $p$ which cannot be connected to $F(p)$ by a $k$-path in $\mathcal{N}(p) \cap X$.

Proof (i) is an immediate consequence of Lemma 1. To proof (ii) consider $p \in D \cap \partial_{k} X$, such that $p$ is not $k$-simple. Then there are at least two different $k$-connected components $A$ and $B$ of $\mathcal{N}(p) \cap X$ which are $k$-adjacent to $p$. Consider $x \in A$ and $y \in B k$ adjacent to $p$. Then, by Lemma 1 , one of them, say $x$, must belong to $D$. Now, if $y \in X \backslash D$, by Lemma $1, F(p) \subset B$ and $x \in D$ cannot be connected to $F(p)$ by a $k$-path in $\mathcal{N}(p) \cap X$. On the other hand, if both $x, y \in D$, since $F(p) \subset \mathcal{N}(p) \cap X$ is $k$-connected, then either $F(p) \cap A=\emptyset$ or $F(p) \cap B=\emptyset$. If $F(p) \cap A=\emptyset$, then $x \in D$ cannot be connected to $F(p)$ by a $k$-path in $\mathcal{N}(p) \cap X$; and if $F(p) \cap B=\emptyset$, then $y \in D$ cannot be connected to $F(p)$ by a $k$-path in $\mathcal{N}(p) \cap X$.

Proof of Theorem 8 By Theorem 7, $\{p, q\}$ is a $k$-simple pair. We show now that $p$ and $q$ must be $k$-simple points. Suppose that $p$ is not $k$-simple. We have two possibilities: $p$ is a $k$ boundary point or $p$ is a $k$-interior point.

Suppose first that $p$ is a $k$-boundary point. Consider $r k$ adjacent to $p$ such that $r \in F(p)$ (this implies that $r \neq q$ ). Since $p$ is not $k$-simple, by Theorem 4 , there exists $s \in X$, $k$-adjacent to $p$, which can not be connected to $r$ by a $k$ path in $\mathcal{N}(p) \cap X$. Suppose that $s \neq q$. Then there are two different $k$-connected components of $\mathcal{N}(p) \cap(X \backslash\{p, q\}) k$ adjacent to $p$, which is impossible by Lemma 1 . Therefore $s=q$ and, as a consequence, $F(p)$ and $q$ lie in different $k$ connected components of $\mathcal{N}(p) \cap X$. This is a contradiction because $F(p) \cup F(q) \subset \mathcal{N}(p, q) \cap X$ is $k$-connected and must be $k$-adjacent to $q$.

Suppose now that $p$ is a $k$-interior point and denote the points in $\mathcal{N}(p, q)$ as in Fig. 17. Then, for $k=4$, $X \supset\left\{p_{1}, p_{2}, p_{3}, p_{7}, p_{8}, p_{9}, p_{10}\right\}$. Since $F(q) \subset \mathcal{N}(q)$ is 4connected and $p_{3}$ and $p_{7}$ are 4-adjacent to (or contained in) $F(q)$, then $\left\{p_{4}, p_{5}, p_{6}\right\} \subset F(q) \subset X$. Then $q$ will also be a 4-interior point and hence $\{p, q\}$ could not be a 4-simple pair. Contradiction.

Analogously, if $k=8$ then $X \supset\left\{p_{2}, p_{8}, p_{10}\right\}$. Since $F(q) \subset \mathcal{N}(q)$ is 8-connected and $p_{2}$ and $p_{8}$ are 8-adjacent to (or contained in) $F(q)$, then $\left\{p_{3}, p_{5}, p_{7}\right\} \subset F(q) \subset X$. But this implies that $q$ is also an 8 -interior point of $X$ and hence $\{p, q\}$ could not be an 8 -simple pair. Contradiction.

The proof of the converse implication is constructive and similar to that of Theorem 5 .

For $k=8$, we have to take into account the fact that an 8 -simple pair of 8 -simple points, neither of them 8 -interior, must be (up to rotation or symmetry) as in Fig. 20, where the white squared points can (or not) belong to $X$.
Fig. 20 An 8-simple pair of 8 -simple points
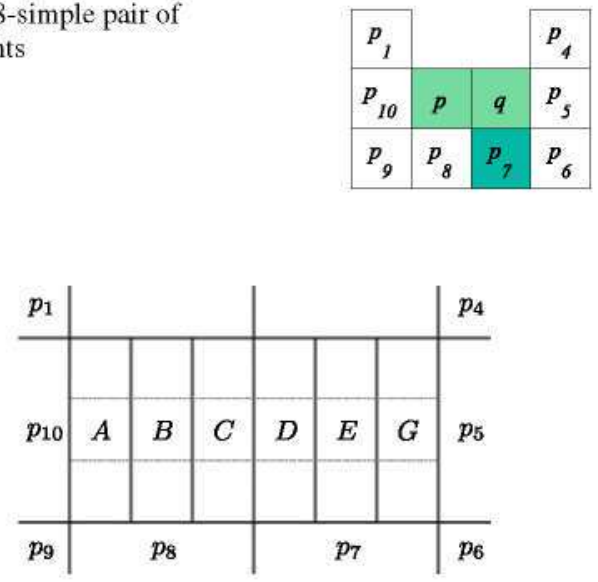

Fig. 21 First subdivision of an 8-simple pair of 8-simple points

Then the multivalued function $F$ defined by $F(x)=\{x\}$ if $x \notin\{p, q\}$ and

$F(p)= \begin{cases}\left\{p_{7}\right\} & \text { if } p_{8} \notin X, \\ \left\{p_{8}\right\} & \text { if } p_{8} \in X, p_{1} \notin X, \\ \left\{p_{8}, p_{10}\right\} & \text { if } p_{1} \in X,\end{cases}$

$F(q)= \begin{cases}\left\{p_{7}\right\} & \text { if } p_{4} \notin X, \\ \left\{p_{5}, p_{7}\right\} & \text { if } p_{4} \in X,\end{cases}$

is 8 -continuous.

To see this consider the first subdivision of $X$ labeled as in Fig. 21.

Then we define $f$, that induces $F$, according to the following table:

\begin{tabular}{lll}
\hline$p_{8} \notin X$ & $f(A)=f(B)=f(C)=p_{7}$ & $p_{4} \notin X$ \\
& $f(D)=f(E)=f(G)=p_{7}$ & \\
$p_{8} \in X$ & $f(A)=f(B)=f(C)=p_{8}$ & \\
$p_{1} \notin X$ & $f(D)=f(E)=f(G)=p_{7}$ & \\
$p_{1} \in X$ & $f(A)=p_{10}, f(B)=f(C)=p_{8}$ & \\
& $f(D)=f(E)=f(G)=p_{7}$ & \\
\hline$p_{8} \notin X$ & $f(A)=f(B)=f(C)=p_{7}$ & $p_{4} \in X$ \\
& $f(D)=f(E)=p_{7}, f(G)=p_{5}$ & \\
$p_{8} \in X$ & $f(A)=f(B)=f(C)=p_{8}$ & \\
$p_{1} \notin X$ & $f(D)=f(E)=p_{7}, f(G)=p_{5}$ & \\
$p_{1} \in X$ & $f(A)=p_{10}, f(B)=f(C)=p_{8}$ & \\
& $f(D)=f(E)=p_{7}, f(G)=p_{5}$ & \\
\hline
\end{tabular}

Observe that $f(p)$ has to be 8 -adjacent to $p$, and $f(q)$ has to be 8 -adjacent to $q$.

On the other hand, a 4-simple pair of 4-simple points (neither of them 8-interior) must be (up to rotation or symmetry) as in Fig. 22, where the white squared points can (or not) belong to $X$. 
Fig. 22 A 4-simple pair $\{p, q\}$ of 4-simple points (neither 8-interior)

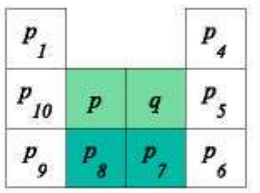

Fig. 23 A 4-simple pair of 4-simple points (one 8 -interior)

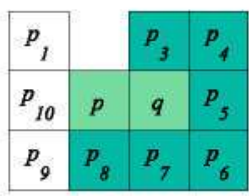

Fig. 24 There exists $(\mathcal{N}, 4)$-retraction but $q$ is not 4-simple

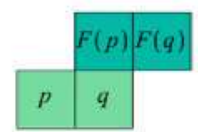

Then the multivalued function $F$ defined by $F(x)=\{x\}$ if $x \notin\{p, q\}$ and

$$
\begin{aligned}
& F(p)= \begin{cases}\left\{p_{8}\right\} & \text { if } p_{10} \notin X, \\
\left\{p_{8}, p_{9}\right\} & \text { if } p_{10} \in X,\end{cases} \\
& F(q)= \begin{cases}\left\{p_{7}\right\} & \text { if } p_{5} \notin X, \\
\left\{p_{6}, p_{7}\right\} & \text { if } p_{5} \in X,\end{cases}
\end{aligned}
$$

is 4-continuous, as can be proved in a similar way as for $k=8$, and $F(p)$ (respectively, $F(q))$ is 4-adjacent to $p$ (respectively, $q$ ).

Finally, if one of the points is 8-interior, as in Fig. 23, where the white squared points can (or not) belong to $X$, then it is not possible to define $F$ in a consistent way because, if we consider any subdivision $X_{r}$ of $X$ and any $f_{r}$ inducing $F$, then the image of the point at the north-west corner in $i_{r}^{-1}(q)$ should be $p_{3}$ or $p_{4}$. Then the image of the point at the north-east corner in $i_{r}^{-1}(p)$ should be $p_{3}, p_{4}$ or $p_{5}$, but it only could be $p_{3}$ since $F(p) \subset \mathcal{N}(p)$. Moreover, since $F(p)$ must be connected, we must have $F(p)=\left\{p_{3}\right\}$. But this contradicts the fact that $F(p)$ must be 4-adjacent to $p$ (and also that the image of the point at the south-west corner of $i_{r}^{-1}(p)$ should be $p_{8}, p_{9}$ or $\left.p_{10}\right)$. This completes the proof of the theorem.

The example of Fig. 24 shows that, without imposing the condition that $F(p) \cup\{p\}$ and $F(q) \cup\{q\} k$-connected, the existence of an $(\mathcal{N}, k)$-retraction $F: X \longrightarrow X \backslash\{p, q\}$, with $\{p, q\}$ a simple pair, does not imply, for $k=4$, that $p$ and $q$ are both 4-simple points of $X$.

\section{$7(\mathcal{N}, k)$-Retractions and $k$-Deletable Sets}

If $D$ is $k$-deletable in $X$, by Theorem 6 , there is an ordering of the points of $D, D=\left\{p_{i}\right\}_{i=1}^{n}$, such that $p_{1}$ is a $k$ -
Fig. 25 A $k$-deletable set not defining an $(\mathcal{N}, k)$-retraction

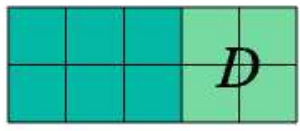

Fig. $26 p$ not 4-simple without $x \in X \backslash D$ 4-adjacent to $p$

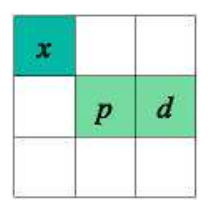

simple point of $X$ and, for $2 \leq i \leq n, p_{i}$ is a $k$-simple point of $X \backslash \bigcup_{j=0}^{i-1}\left\{p_{j}\right\}$. Therefore, by Corollary 7, there exists a $k$-retraction $F: X \rightarrow X \backslash D$. However, a $k$-deletable set does not define in general an $(\mathcal{N}, k)$-retraction, as shown by the example in Fig. 25.

In [8] we gave some partial results related to some wellknown strategies to guarantee the preservation of topology under parallel deletion of simple points.

In this section we address the reciprocal question, i.e., we give conditions under which the existence of an $(\mathcal{N}, k)$ retraction $F: X \rightarrow X \backslash D$ guarantees that $D$ is $k$-deletable.

In particular, we prove the following result:

Theorem 9 Consider $X \subset \mathbb{Z}^{2} k$-connected and let $D$ be a subset of the $k$-boundary of $X$ such that there exists an $(\mathcal{N}, k)$-retraction $F: X \longrightarrow X \backslash D$. Then:

1. If $k=4$, then $D$ is 4-deletable.

2. If $k=8$ and $F(p) \subset \mathcal{N}_{4}(p)$ for every $p \in D$, then $D$ is 8 -deletable and made of 8-simple points.

Proof We will prove first that the hypothesis of the theorem guarantees the existence of a point of $D$ which is $k$-simple in $X$.

Consider first the case $k=4$. Consider any $p \in D$. If $p$ is $k$-simple in $X$ we are done. Suppose that $p$ is not $k$-simple in $X$. Then, we have two possibilities: $p$ is 4-adjacent to $X \backslash D$, or $p$ is not.

Suppose $p$ is not 4-adjacent to $X \backslash D$. Then $F(p)$ is a 4-connected subset of $\mathcal{N}(p) \cap(X \backslash D)$ that is 8-adjacent but not 4-adjacent to $p$, so $F(p)$ must be a single point. Hence $F(p)=\{x\} \subset X \backslash D$ is 8-adjacent but not 4-adjacent to $p$. Since $p$ is not 4-simple, by Theorem 4, there exists $d \in X 4$ adjacent to $p$ which cannot be connected to $x$ by a 4-path in $\mathcal{N}(p) \cap X$. In fact, $d \in D$, because if $d \in X \backslash D$, then $F(p)=$ $\{x\}$ and $F(d)=\{d\}$ would not be 4-adjacent. Therefore $p$, $x$ and $d$ must be situated, up to rotation or symmetry, as in Fig. 26, where the white squared points can (or not) belong to $X$.

Then it is not possible to define $F(d) \subset \mathcal{N}(d)$, such that $F(p) \cup F(d) \supset\{x\}$ is 4-connected, because $\mathcal{N}_{4}(p) \cap X \backslash$ $D=\emptyset$. Contradiction. 

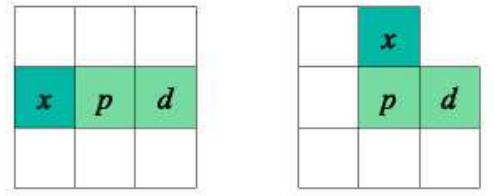

Fig. $27 p$ not 4-simple with $x \in X \backslash D$ 4-adjacent to $p$

Therefore, there is $x \in X \backslash D$ 4-adjacent to $p$. This point $x$ may not be in $F(p)$ but, by Corollary 8 (i), it must be 4-connected in $\mathcal{N}(p) \cap X$ to $F(p)$. Moreover, since $p$ is not 4-simple, then, by Corollary 8 (ii), there exists $d \in D$ 4-adjacent to $p$ which cannot be connected to $x$ by a 4-path in $\mathcal{N}(p) \cap X$. Therefore $p, x$ and $d$ must be situated, up to rotation or symmetry, in one of the configurations of Fig. 27, where the white squared points can (or not) belong to $X$.

In the case on the left, we have the following: $F(p) \cup$ $F(d)$ is 4-connected. Then $F(d) \cup\{d\}$ can not be 4connected (if it were, $d$ would be connected to $x$ by a 4-path in $\mathcal{N}(p) \cap X)$. Therefore, the only possibility for $F(d)$ is one of the points to the north or to the south of $p$. Hence the point to the north or to the south of $p$ must be in $X \backslash D$, so the situation shown on the left reduces to that on the right.

For the case on the right, again $F(p) \cup F(d)$ is 4connected but $F(d) \cup\{d\}$ can not be 4-connected. Therefore, $F(d)$ must be equal to $x$ or to the point to the south of $p$. Consider the points $r$ to the right of $d$ and $s$ to the south of $d$. Suppose that $F(d)=\{x\}$. Then neither $r$ nor $s$ can belong to $X$, because if either of them were in $X$, then its image under $F$ should be 4-adjacent to (or contain) $x$, which is impossible, since $F$ is an $(\mathcal{N}, k)$-retraction. Therefore $d$ is 4-simple. On the other hand, if $F(d)$ is equal to the point to the south of $p$, then $s \notin X$ (if $s \in X$, then $d$ would be 4-connected to $F(d)$, and hence to $F(p)$, and hence to $x$, in $\mathcal{N}(p) \cap X)$. Neither is $r \in X$, because if $r \in X$, then $F(r) \cup F(d)$ should be 4-connected, which is impossible, since $F$ is an $(\mathcal{N}, k)$-retraction. Therefore, $d$ is also 4 -simple in this case.

Suppose now that $k=8$ and $F(p) \subset \mathcal{N}_{4}(p)$ for all $p \in$ $D$. Suppose, for some $p \in D$, that $p$ is not 8 -simple in $X$. Then, by Corollary 8 (ii), there exists $d \in \mathcal{N}(p) \cap D$ which cannot be connected to $F(p)$ by an 8-path in $\mathcal{N}(p) \cap X$. Since $p$ is an 8-boundary point and $F(p) \subset \mathcal{N}_{4}(p), F(p)$ is formed by at most three points. Moreover, if it was formed by three points, then $d$ would be connected to $F(p)$ by a path in $\mathcal{N}(p) \cap X$.

Therefore $F(p)$ is formed by one or two points and, since $d$ cannot be connected to $F(p)$ by a path in $\mathcal{N}(p) \cap X$, we have (up to rotation or symmetry) only three possibilities for $F(p)$ and $d$ displayed in Fig. 28.

Since $p$ and $d$ are 8-adjacent, then $F(p) \cup F(d)$ must be 8 -connected, but this is impossible without connecting $F(p)$ and $d$ by an 8-path in $\mathcal{N}(p) \cap X$. Contradiction.
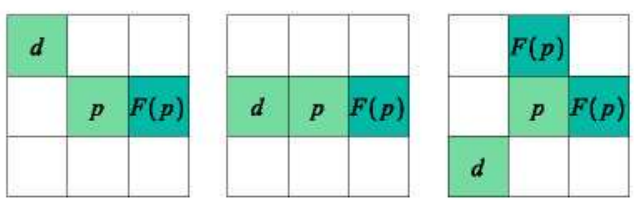

Fig. $28 p$ not 8-simple such that $F(p) \subset \mathcal{N}_{4}(p)$

Therefore, we have proved that the existence of an $(\mathcal{N}, k)$-retraction in the conditions of the theorem guarantees, for $k=4$, the existence of at least a 4-simple point in $D$, and, for $k=8$, that all points of $D$ are 8 -simple.

To prove that $D$ is $k$-deletable we use induction on the cardinal of $D$.

If $D$ consists of just one point, then the result is immediate by Theorem 5 and the definition of a $k$-simple point.

If $D$ consists of more than one point, consider $p \in D k$ simple. Then, we can delete it obtaining $X^{\prime}=X \backslash\{p\}$ and $D^{\prime}=D \backslash\{p\}$ and, since $p$ is $k$-simple in $X$, after deleting it from $X$ :

- no $k$-connected component of $X$ vanishes or is split in several components,

- no $\bar{k}$-connected hole in $X$ is created or is merged with the background or with another such hole.

On the other hand, since $\left.F\right|_{X^{\prime}}: X^{\prime} \longrightarrow X^{\prime} \backslash D^{\prime}=X \backslash D$ is still an $(\mathcal{N}, k)$-retraction, by the induction hypothesis, $D^{\prime}$ is $k$-deletable in $X^{\prime}$, i.e., after deleting $D^{\prime}$ from $X^{\prime}$ :

- no $k$-connected component of $X^{\prime}$ vanishes or is split in several components,

- no $\bar{k}$-connected hole in $X^{\prime}$ is created or is merged with the background or with another such hole.

Therefore, after deleting $D=\{p\} \cup D^{\prime}$ from $X$ :

- no $k$-connected component of $X$ vanishes or is split in several components,

- no $\bar{k}$-connected hole in $X$ is created or is merged with the background or with another such hole,

i.e., $D=D^{\prime} \cup\{p\}$ is $k$-deletable in $X$. This ends the proof of the theorem.

The proof of the previous results depends on the existence of a simple point in $D$.

The example on the left of Fig. 29 (see also Fig. 24) shows that, in the hypothesis of the theorem, for $k=4$, not all the points need to be simple.

On the other hand, the example on the right shows that for $k=8$ the result is no longer true if we do not require that $F(p) \subset \mathcal{N}_{4}(p)$. The reason is that in this case, not all points of $D$ have to be 8 -simple. If we add this condition to Theorem 9 we obtain the following result: 

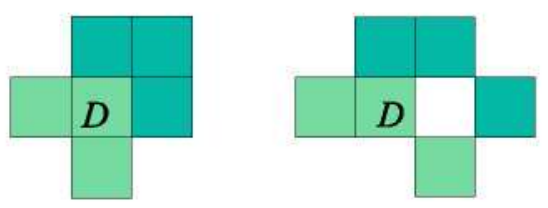

Fig. 29 There exists $(\mathcal{N}, k)$-retraction and: $D$ 4-deletable but not all points of $D$ are 4-simple (left), D is not 8-deletable (right)
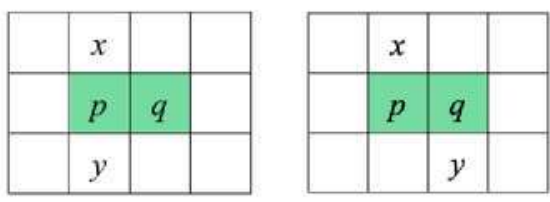

Fig. $30 p, q \in D$ 8-simple in $X, q$ not 8-simple in $X \backslash\{p\}$ with $x, y \in X$ in different 8-connected components of $\mathcal{N}(q) \cap(X \backslash\{p\})$

Theorem 10 Consider $X \subset \mathbb{Z}^{2}$ and let $D$ be a set of $k$-simple points of $X$ such that there exists an $(\mathcal{N}, k)$ retraction $F: X \longrightarrow X \backslash D$. Then $D$ is $k$-deletable.

Proof For $k=4$ it is an immediate consequence of the previous theorem.

For $k=8$, we will prove that if we delete any $p \in D$, then the points of $D \backslash\{p\}$ are 8 -simple points of $X \backslash\{p\}$.

Consider any $p \in D$. Suppose that, after deleting $p$, there exists $q \in D \backslash\{p\}$ which is not 8 -simple in $X \backslash\{p\}$. Then, $q$ is an 8-boundary point of $X$ such that, before deleting $p$, the number of 8-connected components of $\mathcal{N}(q) \cap X$ which were 8 -adjacent to $q$ was equal to 1 and, after deleting $p$ the number of 8-connected components of $\mathcal{N}(q) \cap X \backslash\{p\}$ is different from one. If that number were 0 this would imply that $p$ is the only point 8 -adjacent to $q$, but then $F(p)$ and $q$ would be in two different 8 -connected components of $\mathcal{N}(p) \cap X$, which is impossible because $p$ is 8 -simple.

Therefore, $p$ and $q$ are 8 -adjacent points such that there are two 8-connected components of $\mathcal{N}(q) \cap(X \backslash\{p\})$ which are 8 -adjacent to $q$. Moreover, if $p$ and $q$ were 8 -adjacent but not 4-adjacent, the deletion of $p$ would not affect the 8connectivity of $\mathcal{N}(q) \cap X$. This is due to the fact that the deletion of a corner point of an 8-connected component of $\mathcal{N}(q) \cap X$ can not split it in two components.

Therefore $p$ and $q$ are situated (up to rotation or symmetry) as in Fig. 30, where $x, y \in X$ are points in different 8-connected components of $\mathcal{N}(q) \cap(X \backslash\{p\})$.

In the figure on the left, the point to the left of $p$ cannot belong to $X$ because $p$ cannot be an 8 -interior point of $X$. On the other hand, if in the figure on the right, either of the points south of $p$ or north of $q$ were in $X$ then its analysis would reduce to the case on the left. Therefore, we can suppose that neither of them belongs to $X$. Therefore, $p$, $q, x$ and $y$ are situated (up to rotation or symmetry) as in Fig. 31 .
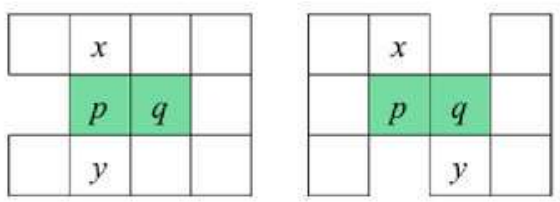

Fig. $31 p, q \in D$ 8-simple in $X, q$ not 8-simple in $X \backslash\{p\}$ with $x, y \in X$ in different 8-connected components of $\mathcal{N}(q) \cap(X \backslash\{p\})$

For the figure on the left, $F(p) \cup F(x)$ and $F(p) \cup F(y)$ cannot both be 8 -connected, since $F$ is an $(\mathcal{N}, 8)$-retraction.

For the figure on the right, since $F(p) \cup F(q)$ must be 8connected, either $F(p)=\{y\}$ or $F(q)=\{x\}$. But if $F(p)=$ $\{y\}$, then $F(p) \cup F(x)$ cannot be 8-connected, and if $F(q)=$ $\{x\}$, then $F(q) \cup F(y)$ cannot be 8 -connected.

In every case we get a contradiction. Hence, for every $p \in D, q$ is 8 -simple in $X \backslash\{p\}$.

Now, the result follows, as in Theorem 9, using induction on the cardinal of $D$, as follows:

If $D$ consists of just one point, then the result is immediate.

If $D$ consists of more than one point, consider any 8simple point $p \in D$. Then, we can delete it obtaining $X^{\prime}=$ $X \backslash\{p\}$ and $D^{\prime}=D \backslash\{p\}$ such that $D^{\prime}$ is a set of 8-simple points of $X^{\prime}$ such that $\left.F\right|_{X^{\prime}}: X^{\prime} \longrightarrow X^{\prime} \backslash D^{\prime}=X \backslash D$ is still an $(\mathcal{N}, 8)$-retraction. Therefore, by induction hypothesis, $D^{\prime}$ is 8-deletable in $X^{\prime}$ and, by an argument similar to that of Theorem 9, $D=D^{\prime} \cup\{p\}$ is 8 -deletable in $X$.

This ends the proof of the theorem.

Remark 5 Alternatively, Theorem 10 can be proved showing that if $D$ is a set of 8 -simple points of $X$ such that there exists an $(\mathcal{N}, 8)$-retraction $F: X \longrightarrow X \backslash D$, then $D$ satisfies Ronse sufficient conditions for a set $D \subset X$ to be 8deletable in $X$ (see, for example, [13]), which can be stated as follows:

R1. All points of $D$ are 8-simple.

R2. For every two 4-adjacent points $p, q \in D,\{p, q\}$ is an 8 -simple pair.

R3. $D$ does not contain any 8-component of $X$ formed by 2,3 , or 4 mutually 8 -adjacent points.

However, for $k=4$, if $D$ is a set of 4-simple points of $X$ such that there exists an $(\mathcal{N}, 4)$-retraction $F: X \longrightarrow X \backslash D$, even though that we have seen that $D$ is 4-deletable, $D$ does not necessarily have to satisfy Ronse conditions, which for $k=4$ can be stated as follows:

R1. All points of $D$ are 4-simple.

R2. For every two 8-adjacent points $p, q \in D, p$ (respectively, $q$ ) is 4-simple in $X \backslash\{q\}$ (respectively, $X \backslash\{p\}$ ).

The reason is that a deletable set $D \subset X$ may have points with the local configuration (up to symmetry) of Fig. 32, where $p, q, s \in D, t \in X \backslash D$ and the white squared points can (or not) belong to $X$. 
Fig. $32\{p, q\}$ does not satisfy Ronse conditions for $k=4$

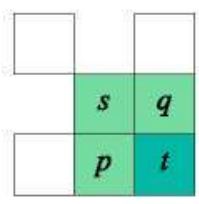

In this case, the pair $\{p, q\}$ does not satisfy Ronse conditions because $q$ is not 4-simple in $X \backslash\{p\}$.

\section{Conclusions and Future Work}

We have presented in this paper a theory of continuity in digital spaces, using multivalued functions, which extends the one introduced by Rosenfeld and provides a framework to define topological notions in a far more realistic way than by using just single-valued digitally continuous functions.

In particular, we have shown that the basic morphological operations of dilation and closing are continuous functions and that, although the dual operations of erosion and opening cannot be modeled as continuous functions on the set of black pixels, they are so if we consider them defined on the set of white pixels.

On the other hand, the deletion of simple points and simple pairs can be completely characterized in terms of digitally continuous multivalued functions. This fact has been used to characterize thinning algorithms in terms of digitally continuous multivalued functions. We have shown that the existence of an $(\mathcal{N}, k)$-retraction $F: X \longrightarrow X \backslash D$ guarantees that $D$ is 4-deletable (respectively, 8 -deletable) whenever $D$ is made of 4-boundary (respectively, 8-simple) points. The converse is not true in general although it holds in certain particular important cases which are at the basis of many thinning algorithms.

The notion and main properties of a digitally continuous multivalued function are presented for any dimension. The results on retractions at the end of Sect. 3 are stated for $\mathbb{Z}^{2}$, since their main role is that of justification of the appropriateness of our notion of continuity, although they are easily extendable to higher dimension. The same is true for the results on mathematical morphology. Several of the results in this paper may be regarded as tools for thinning algorithms for images in $\mathbb{Z}^{2}$. We are working on analogous results for images in $\mathbb{Z}^{3}$.

Finally, we are also working on the design of new parallel thinning algorithms based on the previous construction of an $(\mathcal{N}, k)$-retraction.

Acknowledgements The authors thank the referees for helpful comments and suggestions which have helped to improve the final version of the paper.

Antonio Giraldo has been supported by Ministerio de Ciencia e Innovación (MICINN MTM2006-0825 and MTM2009-07030). All authors have been supported by Comunidad Autónoma de Madrid and Universidad Po litécnica de Madrid (UPM-CAM Q061010133).

\section{References}

1. Boxer, L.: Digitally continuous functions. Pattern Recognit. Lett. 15, 833-839 (1994)

2. Boxer, L.: A classical construction for the digital fundamental group. J. Math. Imaging Vis. 10, 51-62 (1999)

3. Boxer, L.: Properties of digital homotopy. J. Math. Imaging Vis. 22, 19-26 (2005)

4. Boxer, L.: Homotopy properties of sphere-like digital images. J. Math. Imaging Vis. 24, 167-175 (2006)

5. Chen, L.: Gradually varied surface and its optimal uniform approximation. Proc. SPIE 2182, 300-307 (1994)

6. Chen, L.: Discrete Surfaces and Manifolds: A Theory of DigitalDiscrete Geometry and Topology. Scientific and Practical Computing, Rockville (2004)

7. Escribano, C., Giraldo, A., Sastre, M.A.: Digitally continuous multivalued functions. In: Coeurjolly, D., Sivignon, I., Tougne, L., Dupont, F. (eds.) Discrete Geometry for Computer Imagery 2008. Lecture Notes in Computer Science, vol. 4992, pp. 81-92. Springer, Berlin (2008)

8. Escribano, C., Giraldo, A., Sastre, M.A.: Thinning algorithms as multivalued $\mathcal{N}$-retractions. In: Brlek, S., Reutenauer, C., Provençal, X. (eds.) Discrete Geometry for Computer Imagery 2009. Lecture Notes in Computer Science, vol. 5810, pp. 275287. Springer, Berlin (2009)

9. Giraldo, A., Gross, A., Latecki, L.J.: Digitizations preserving shape. Pattern Recognit. 32, 365-376 (1999)

10. Giraldo, A., Sanjurjo, J.M.R.: Density and finiteness. A discrete approach to shape. Topol. Appl. 76, 61-77 (1997)

11. Han, X., Xu, C., Prince, J.: A topology preserving level set method for geometric deformable models. IEEE Trans. Pattern Anal. Mach. Intell. 25(6), 755-768 (2003)

12. Khalimsky, E.: Topological structures in computer science. J. Appl. Math. Simul. 1, 25-40 (1987)

13. Klette, R., Rosenfeld, A.: Digital Geometry. Elsevier, Amsterdam (2004)

14. Kong, T.Y.: A digital fundamental group. Comput. Graph. 13, 159-166 (1989)

15. Kong, T.Y., Rosenfeld, A.: Digital topology: introduction and survey. Comput. Vis. Graph. Image Process. 48, 357-393 (1989)

16. Kong, T.Y., Rosenfeld, A. (eds.): Topological Algorithms for Digital Image Processing. Elsevier, Amsterdam (1996)

17. Kovalevsky, V.: A new concept for digital geometry. In: Ying-Lie, O., et al. (eds.) Shape in Picture. Proc. of the NATO Advanced Research Workshop, Driebergen, The Netherlands, 1992. Computer and Systems Sciences, vol. 126. Springer, Berlin (1994)

18. Ronse, C.: A topological characterization of thinning. Theor. Comput. Sci. 43, 31-41 (1988)

19. Rosenfeld, A.: Continuous functions in digital pictures. Pattern Recognit. Lett. 4, 177-184 (1986)

20. Rosenfeld, A.: Digital topology. Am. Math. Mon. 86(8), 621-630 (1979)

21. Ségonne, F.: Active contours under topology control-genus preserving level sets. Int. J. Comput. Vis. 79(2), 107-117 (2008)

22. Soille, P.: Morphological operators. In: Jähne, B., et al. (eds.) Signal Processing and Pattern Recognition. Handbook of Computer Vision and Applications, vol. 2, pp. 627-682. Academic Press, San Diego (1999)

23. Tsaur, R., Smyth, M.B.: "Continuous" multifunctions in discrete spaces with applications to fixed point theory. In: Bertrand, G., et al. (eds.) Digital and Image Geometry: Advanced Lectures. Lecture Notes in Computer Science, vol. 2243, pp. 75-88. Springer, New York (2001) 


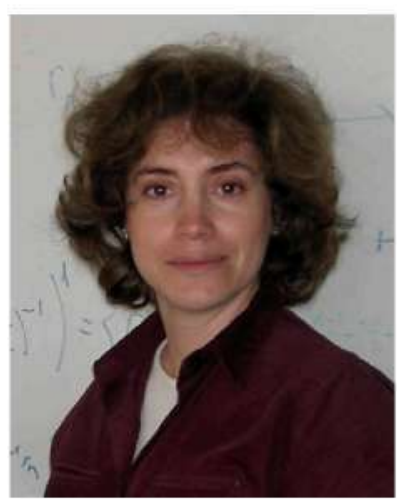

Carmen Escribano received a Bachelor's degree in Mathematics from the Autonoma University of Madrid (Spain)), and she is currently doing her Ph.D. at the Technical University of Madrid (Spain). She is currently a lecturer at the Computer Science School of the Technical University of Madrid. Her current research interests include Orthogonal Polynomials, Operator Theory, Fractal Geometry and Digital Topology and applications.

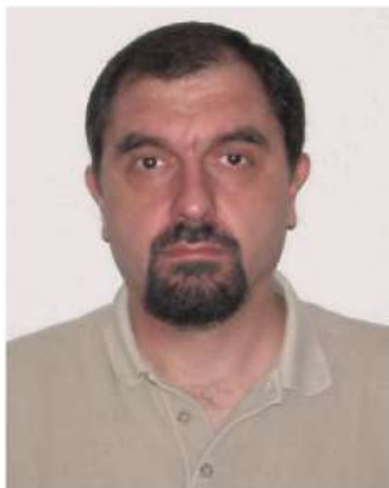

Antonio Giraldo received a Bachelor's degree in Mathematics, and a $\mathrm{Ph} . \mathrm{D}$. Degree in Mathematics from the Complutense University of Madrid (Spain). He is currently an associate professor, and past chair, of the Applied Mathematics Department, at the Computer Science School of the Technical University of Madrid. His current research interests include Topology and it applications to Digital Images and Dynamical Systems, and also Orthogonal Polynomials.

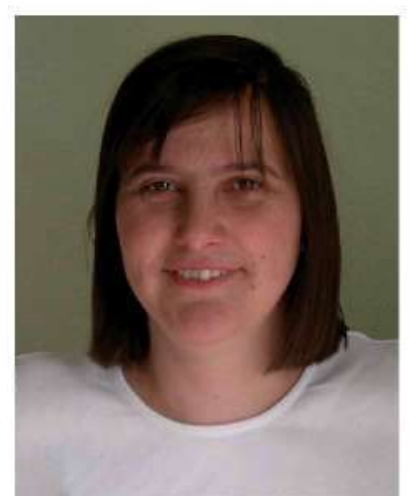

María Asunción Sastre received a Bachelor's degree in Mathematics from the Complutense University of Madrid (Spain), and a Ph.D. Degree from the Technical University of Madrid (Spain). She is currently a senior lecturer at the Computer Science School of the Technical University of Madrid. Her current research interests include Orthogonal Polynomials, Fractal Geometry and Digital Topology and applications. 